\title{
Is set-shifting and central coherence in anorexia nervosa influenced by body mass index, anxiety or depression? A systematic review
}

Tone Seim Fuglset

\begin{abstract}
Background: Anorexia nervosa (AN) is a severe eating disorder, recognized by a relentless pursuit for thinness and extreme low body weight. The disorder is often accompanied by comorbid disorders such as anxiety and depression, and altered neuropsychological function in terms of poor set-shifting and reduced central coherence. The aim of this review was to evaluate whether neuropsychological impairments in AN are influenced by body mass index, anxiety or depression.

Method: A systematic review approach was used, following the PRISMA guidelines for systematic reviews. Literature was identified via searches in PubMed, Psychlnfo and Embase database, by using the search words [anorexia nervosa] AND [central coherence], and [anorexia nervosa] AND [set-shifting]. Studies were included if they were written in English, peer-reviewed, included individuals with AN, included tests measuring setshifting and/or central coherence, investigated associations between set-shifting/central coherence with anxiety and/or depression and/or BMI. Risk of bias was assessed by using a critical appraisal checklist from the Joanna Briggs Institute. Results were summarized in a narrative synthesis.

Results: Although results are heterogeneous, the majority of studies report that neither body mass index (BMI), anxiety or depression is associated with altered central coherence and set-shifting in individuals with AN.

Conclusions: Findings indicate that BMI, depression and anxiety does not influence neuropsychological function in AN, suggesting that it could be a characteristic of the disorder. A complete understanding of predisposing, precipitating and maintaining factors in AN needs to be addressed in future research. This could contribute to the development of better and more targeted treatment strategies.
\end{abstract}

Keywords: Anorexia nervosa, Set-shifting, Central coherence, Anxiety, Depression, Body mass index, Systematic review

Correspondence: Tone.Seim.Fuglset@helse-mr.no

Møre and Romsdal Hospital Trust, Molde Hospital, Parkvegen 84, 6412 Molde,

Norway

(c) The Author(s). 2021 Open Access This article is licensed under a Creative Commons Attribution 4.0 International License, which permits use, sharing, adaptation, distribution and reproduction in any medium or format, as long as you give appropriate credit to the original author(s) and the source, provide a link to the Creative Commons licence, and indicate if changes were made. The images or other third party material in this article are included in the article's Creative Commons licence, unless indicated otherwise in a credit line to the material. If material is not included in the article's Creative Commons licence and your intended use is not permitted by statutory regulation or exceeds the permitted use, you will need to obtain permission directly from the copyright holder. To view a copy of this licence, visit http://creativecommons.org/licenses/by/4.0/ The Creative Commons Public Domain Dedication waiver (http://creativecommons.org/publicdomain/zero/1.0/) applies to the data made available in this article, unless otherwise stated in a credit line to the data. 


\section{Background}

Eating disorders are characterized by a persistent disturbance of eating or eating-related behaviour that result in altered consumption of food, which significantly impairs physical health or psychosocial functioning [1]. Anorexia nervosa (AN) is a severe eating disorder, with the highest mortality rate of any mental illness [2]. It is recognized by a relentless pursuit for thinness, extreme low body weight, and is often accompanied by a disturbed body image and a denial of illness. The aetiology of AN remains unclear, however the disorder is most likely caused by a complex interaction between genetic and environmental factors. Genetic variants significantly associated with AN have recently been identified, which demonstrates that the origins of this disorder is both metabolic and psychiatric [3].

Neuropsychological function in AN has been investigated in a number of studies, suggesting that specific neuropsychological impairments may be related to the pathophysiology of the disorder. These studies show that individuals with AN often display poor set-shifting abilities and weak central coherence (for systematic reviews see $[4,5])$. Set-shifting, or cognitive flexibility, refers to the ability to shift thoughts or actions according to situational demands. Central coherence is the degree to focus on details in processing information and the global integration of such information [6]. Individuals with weak central coherence have a preference for details over global processing. These findings are most commonly seen in adults, and findings from studies including adolescents are less consistent $[7,8]$. It is worth noting that adolescence is a time of considerable development of the brain and cognitive abilities, affecting the development of both executive functions and social cognition [9].

Neuropsychological impairments in AN could be a characteristic of the disorder, however, it could also be a consequence of malnutrition and underweight. Yet, the role of starvation is still unclear, as some neuropsychological functions remains intact and general intelligence is within the normal range [10]. In fact, studies have shown that patients with AN perform better on selected tasks, such as tasks requiring local information processing [5, 11, 12]. A review and meta-analysis state that individuals with AN score above the average intelligence quotient of the normative population in the National Adult Reading Test and Wechsler Intelligence Scales [13].

Individuals with $\mathrm{AN}$ often suffer from comorbid mental disorders. The most common comorbidities are mood disorders, personality disorders, anxiety disorders, obsessivecompulsive disorders, and developmental disorders (e.g. autism spectrum, attention-deficit hyperactivity disorder) [14]. Both depression and anxiety have been linked to cognitive impairments in the domains of episodic memory, verbal memory as well as varying subsets of executive function $[15,16]$. However, the role of depression and anxiety on neuropsychological performance in individuals with AN remains unclear. On one hand, comorbid psychiatric symptoms such as depression and anxiety may contribute to poorer performance on neuropsychological tasks. On the other hand, poorer performance could also be a characteristic of the disorder, and unrelated to noneating disorder psychopathology.

A complete understanding of neuropsychological function in AN may contribute to the development of better and more specific treatment strategies. It would be beneficial to determine whether neuropsychological impairments are related to comorbid disorders or as a consequence of underweight, or whether these are more likely a characteristic of the disorder per se. If so, this should be targeted in treatment.

Set-shifting and central coherence are the most studied cognitive domains in individuals with AN. This systematic review provide an overview of relevant studies and their findings concerning the relationship between clinical measures and neuropsychological function in AN. The aim of the current systematic review is to evaluate whether the observed alterations in set-shifting and central coherence in patients with $\mathrm{AN}$ are influenced by body mass index (BMI), ED severity and broader transdiagnostic factors such as anxiety and depression.

\section{Methods}

This systematic review was guided by the PRISMA criteria [17].

\section{Eligibility criteria}

All primary studies were peer-reviewed and written in English. There were no limitations in terms of time frame. Studies were included if they met the following inclusion criteria:

1. Manuscript written in English

2. Study published in peer-reviewed journals

3. Included individuals diagnosed with $A N$

4. Included tests measuring set-shifting and/or central coherence

5. Investigated associations between set-shifting/central coherence with anxiety and/or depression and/ or BMI

\section{Search strategy and selection of studies}

Literature search was performed in PubMed, PsychInfo and Embase databases. A search for [anorexia nervosa] AND [central coherence] resulted in 73 (PubMed), 54 (PsychInfo) and 73 (Embase) articles. A 
search for [anorexia nervosa] and [set shifting] resulted in 92 (Pubmed), 101 (PsychInfo) and 117 (Embase) articles. See Additional file 1 for search strategy in PubMed. In total, the search resulted in 510 articles. After the initial searches in the different databases, duplicates were removed. Then, all titles and abstracts of 189 articles were examined and evaluated for relevance, which resulted in removal of 76 articles. Then, full text for all potential relevant articles (113), were obtained and screened thoroughly for relevance. Seventy-two articles were then removed due to lack of statistical analyses between neuropsychological function and clinical measures, or they were not relevant for the current review. In all, a total of 41 articles were included in the present study. Figure 1 illustrates a PRISMA flow chart of the search strategy (see Fig. 1).

\section{Data extraction}

The following variables were extracted from the articles: Authors, publication year, sample (size and diagnosis), neuropsychological test, measures of anxiety, depression and eating disorder symptoms, cognitive domain (setshifting or central coherence), medical treatment. Outcome measures included analyses examining the relationship between set-shifting and/or central coherence and depression/anxiety/BMI/weight for height/eating disorder symptoms.

\section{Assessment of risk of bias in included studies}

Risk of bias of the included studies was assessed by using a critical appraisal checklist for case control studies and cross sectional studies from the Joanna Briggs Institute (https://joannabriggs.org/critical-appraisal-tools), which purpose is to assess the methodological quality of a

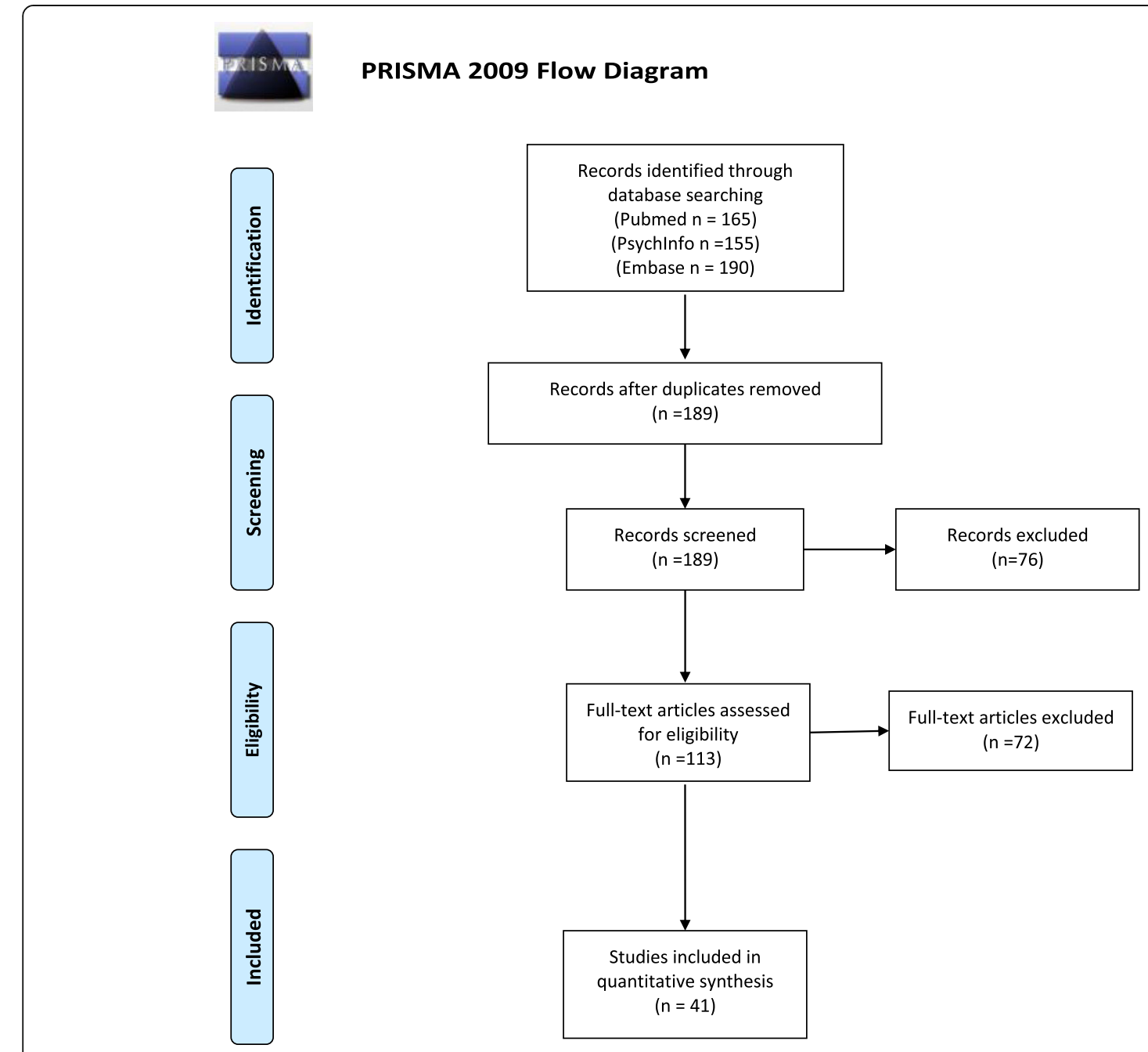

Fig. 1 PRISMA 2009 flow diagram 
study and to determine the extent to which a study has addressed the possibility of bias in its design, conduct and analysis. See Additional files 2 and 3.

\section{Data synthesis}

Due to the heterogeneity of the primary studies, especially considering the wide variety of neuropsychological tests and measures of psychopathology, and that different statistical methods have been used, a narrative synthesis approach was used for this current review.

\section{Study characteristics}

Seven studies included an adolescent sample (age $<18$ years) [18-24], and four studies included both adults and adolescents [25-28]. The remaining 26 studies included an adult sample (age $\geq 18$ years).

A variety of tests were used to measure set-shifting: Haptic Illusion Task, Cognitive Shift Task, Trail Making Test, Brixton Spatial Anticipation Test, Picture Set Test, Verbal Fluency Task, CatBat Task, Intra/Extra Dimensional Set Shift Test, Wisconsin Card Sorting Test, Visual Set-Shifting Task, Berg's Card Sorting Test, Parametric Go/NoGo Test, Category Learning Task, Task Switching Paradigm, Design Fluency Task, Cued Color-Shape Switching Task and Comprehensive Trail Making Test. The following tests were used to measure central coherence: Rey Complex Figure Test, Embedded Figures Test, Sentence Completion Task, Homograph Reading Task, Overlapping Figures Test, Fragmented Pictures Task, Block Design, Object Assembly and Group Embedded Figures Test.

Different measures were used to assess anxiety and depression. The following measures were used to measure anxiety: State-Trait Anxiety Inventory $(n=14)$, Hospital Anxiety and Depression Scale $(n=8)$, Beck's Anxiety inventory $(n=1)$, Depression, Anxiety and Stress Scale $(n=2)$, Spence Children's Anxiety Scale $(n=1)$ and Beck Youth Inventory $(n=1)$. The most common measure of depression was Beck's Depression Inventory $(n=18)$, then HADS $(n=8)$, Depression, Anxiety and Stress Scale $(n=2)$, Hopkins Symptoms Checklist $(n=1)$, Quick Inventory of Depressive Symptomatology, Children's Depression Inventory $(n=1)$ and German depression inventory for children and adolescents $(n=1)$.

To examine the associations between cognitive performance and clinical characteristics, different regression analyses accounting for covariates as well as correlation analyses, both Pearson and Spearman's, were used.

\section{Results}

Table 1 summarises the studies in more detail, including author, sample size (mean age), tests and significant correlations. The studies are organized chronologically according to year of publication. Measure of effect size is not included in the table, as the majority of the studies did not report effect size measures of the association between neuropsychological function and clinical measures.

\section{Risk of bias in included studies}

The majority of the studies included in the review had a low risk of bias, i.e. the groups were comparable other than the presence of disease, and the groups were properly matched. Valid, standardized tests were used, and testing procedures were the same for all participants. Confounding variables were identified and controlled for, and appropriate statistical tests were used. However, in some studies, issues concerning representativeness of the patient group and lacking control of possible confounding factors, led to an increased risk of bias. In one study, there was a low participation rate, which could affect the representativeness of the AN group [53]. Another study included a specific sample of AN with strict inclusion criteria, suggesting that this group was not representative for the AN population [55]. One study had a very heterogeneous AN group in terms of differences in duration of illness, age of onset, treatment experience and different levels of illness severity [50]. Furthermore, some of the studies did not control adequately for confounding factors. Most commonly was not considering the effect of pharmacological treatment on test performance $[18,39,54,56,57]$, and lack of controlling for intelligence quotient $[35,51]$. Other potential confounding factors are comorbidity, which was not assessed and controlled for in several studies [44, 56, 57]. These factors contribute to an increased risk of bias in these studies.

\section{Body mass index and neuropsychological function}

The studies that included associations between BMI and neuropsychological function show variable results. The majority of studies $(n=28)$ found no significant associations between set-shifting or central coherence and BMI [20, 22-24, 27-32, 34-36, 39-45, 48, 52-58]. Some studies $(n=7)$ found an association between BMI and neuropsychological function. One study found that BMI correlated with the number of errors in the Homograph Reading Task, a measure of central coherence [5]. A negative correlation was detected between BMI and the Fragmented Picture Task, but the correlation was not significant after correcting for multiple comparisons [26]. Another study found that significant low central coherence index became non-significant when adjusting for nadir BMI [50]. Herbrich et al. [21] found a negative correlation between BMI and Group Embedded Figures Test in the AN-binge/purge group. In addition, a negative correlation was found between BMI and Trail Making Test -4 in the $\mathrm{AN}$ restrictive group. Studies 
Table 1 Overview of associations between neuropsychological function and clinical measures in patients with AN. Significant associations are highlighted in bold

\begin{tabular}{|c|c|c|c|c|c|c|}
\hline Authors & $\begin{array}{l}\text { Sample } \\
N \text { (mean } \\
\text { age) }\end{array}$ & Test & Anxiety & Depression & BMI/WfH & ED severity \\
\hline $\begin{array}{l}\text { Tchanturia } \\
\text { et al., } 2002 \\
\text { [29] }\end{array}$ & $\begin{array}{l}\mathrm{AN}=30 \\
(25.2) \\
\mathrm{AN}- \\
\mathrm{REC}=16 \\
(30.0) \\
\mathrm{HC}=23 \\
(27.6)\end{array}$ & $\begin{array}{l}\text { Haptic Illusion } \\
\text { Task (SS) } \\
\text { Cognitive Shift } \\
\text { Task (SS) }\end{array}$ & $\begin{array}{l}\text { Group differences in the } \\
\text { Haptic Illusion Task } \\
\text { remained after covarying } \\
\text { for anxiety. Group } \\
\text { differences in the } \\
\text { cognitive shift task } \\
\text { disappeared when } \\
\text { controlling for anxiety. }\end{array}$ & - & N/A & N/A \\
\hline $\begin{array}{l}\text { Tchanturia } \\
\text { et al., } 2004 \\
\text { [30] }\end{array}$ & $\begin{array}{l}\mathrm{AN}=34 \\
(26.7) \\
\mathrm{BN}=19 \\
(26.5) \\
\mathrm{HC}=35 \\
(24.8)\end{array}$ & $\begin{array}{l}\text { Trail Making Test } \\
\text { (SS) } \\
\text { Brixton Spatial } \\
\text { Anticipation Test } \\
\text { (SS) } \\
\text { Picture Set Test } \\
\text { (SS) } \\
\text { Verbal Fluency } \\
\text { Task (SS) } \\
\text { CatBat Task (SS) } \\
\text { Haptic Illusion } \\
\text { Task (SS) }\end{array}$ & - & - & - & N/A \\
\hline $\begin{array}{l}\text { Holliday } \\
\text { et al., } 2005 \\
{[31]}\end{array}$ & $\begin{array}{l}\mathrm{AN}=47 \\
(26.3) \mathrm{AN} \\
\text { sisters }= \\
47(27.6) \\
\mathrm{HC}=47 \\
(26.5)\end{array}$ & $\begin{array}{l}\text { Haptic Illusion } \\
\text { Task (SS) } \\
\text { Brixton Spatial } \\
\text { Anticipation Test } \\
\text { (SS) } \\
\text { Trail Making Test } \\
\text { (SS) } \\
\text { CatBat Task (SS) }\end{array}$ & $\begin{array}{l}\text { Anxiety scores correlated } \\
\text { with the Haptic Illusion } \\
\text { Task. }\end{array}$ & $\begin{array}{l}\text { Depression scores } \\
\text { correlated with the } \\
\text { Haptic Illusion Task. }\end{array}$ & - & N/A \\
\hline $\begin{array}{l}\text { Fowler } \\
\text { et al., } 2006 \\
\text { [20] }\end{array}$ & $\begin{array}{l}\mathrm{AN}=25 \\
(16.9) \\
\mathrm{HC}=25 \\
(17.6)\end{array}$ & $\begin{array}{l}\text { Intra/Extra } \\
\text { Dimensional Set } \\
\text { Shift Test (SS) }\end{array}$ & - & - & - & N/A \\
\hline $\begin{array}{l}\text { Steinglass } \\
\text { et al., } 2006 \\
{[32,33]}\end{array}$ & $\begin{array}{l}\mathrm{AN}=15 \\
(25.6) \\
\mathrm{HC}=11 \\
(24.0)\end{array}$ & $\begin{array}{l}\text { Wisconsin Card } \\
\text { Sorting Test (SS) }\end{array}$ & - & - & - & - \\
\hline $\begin{array}{l}\text { Lopez et al., } \\
2008 \text { [5] }\end{array}$ & $\begin{array}{l}\mathrm{AN}=42 \\
(28.4) \\
\mathrm{HC}=42 \\
(26.3)\end{array}$ & $\begin{array}{l}\text { Rey Complex } \\
\text { Figure Test (CC) } \\
\text { Embedded } \\
\text { Figures Test (CC) } \\
\text { Sentence } \\
\text { Completion Task } \\
\text { (CC) } \\
\text { Homograph } \\
\text { Reading Task } \\
\text { (CC) }\end{array}$ & - & - & $\begin{array}{l}\text { BMI correlated with } \\
\text { number of errors in the } \\
\text { initial pronunciation in } \\
\text { the Homograph Reading } \\
\text { Task. }\end{array}$ & N/A \\
\hline $\begin{array}{l}\text { Kim et al., } \\
2010 \text { [34] }\end{array}$ & $\begin{array}{l}\mathrm{AN}=40 \\
(22.8) \\
\mathrm{BN}=28 \\
(23.0) \\
\mathrm{HC}=34 \\
(22.7)\end{array}$ & $\begin{array}{l}\text { Trail Making Test } \\
\text { (SS) }\end{array}$ & - & $\begin{array}{l}\text { Depression slightly } \\
\text { correlated with set- } \\
\text { shifting. }\end{array}$ & - & - \\
\hline $\begin{array}{l}\text { Roberts } \\
\text { et al., } 2010 \\
{[35]}\end{array}$ & $\begin{array}{l}\mathrm{AN}=35 \\
(23.7) \\
\mathrm{AN}-\mathrm{BP}= \\
33(25.6) \\
\mathrm{BN}=30 \\
(26.4) \\
\mathrm{AN}- \\
\mathrm{REC}=30 \\
(32.1)\end{array}$ & $\begin{array}{l}\text { Trail Making Test } \\
\text { (SS) } \\
\text { Wisconsin Card } \\
\text { Sorting Test (SS) } \\
\text { Brixton Spatial } \\
\text { Anticipation Test } \\
\text { (SS) } \\
\text { Haptic illusion } \\
\text { Task (SS) }\end{array}$ & $\begin{array}{l}\text { Increased anxiety in the } \\
\text { poor set-shifting group, } \\
\text { but effects were small }\end{array}$ & $\begin{array}{l}\text { Increased depression in } \\
\text { the poor set-shifting } \\
\text { group, but effects were } \\
\text { small. }\end{array}$ & - & $\begin{array}{l}\text { Those with } \\
\text { poor set- } \\
\text { shifting had } \\
\text { higher score } \\
\text { on the YBC- } \\
\text { EDS }\end{array}$ \\
\hline
\end{tabular}


Table 1 Overview of associations between neuropsychological function and clinical measures in patients with AN. Significant associations are highlighted in bold (Continued)

\begin{tabular}{|c|c|c|c|c|c|c|}
\hline Authors & $\begin{array}{l}\text { Sample } \\
N \text { (mean } \\
\text { age) }\end{array}$ & Test & Anxiety & Depression & BMI/WfH & ED severity \\
\hline & $\begin{array}{l}\text { AN } \\
\text { sister = } \\
30(24.2) \\
\text { BN } \\
\text { sister = } \\
20(27.6) \\
\text { HC = 88 } \\
(28.4)\end{array}$ & & & & & \\
\hline $\begin{array}{l}\text { Tenconi } \\
\text { et al., } 2010 \\
{[36]}\end{array}$ & $\begin{array}{l}\text { AN }=153 \\
(26.2) \\
H C=120 \\
(27.4)\end{array}$ & $\begin{array}{l}\text { Wisconsin Card } \\
\text { Sorting Test (SS) } \\
\text { Trail Making Test } \\
\text { (SS) } \\
\text { Rey Complex } \\
\text { Figure Test CC) } \\
\text { Overlapping } \\
\text { Figures Test (CC) }\end{array}$ & $\begin{array}{l}\text { State anxiety had a } \\
\text { moderate correlation with } \\
\text { Trail Making Test and } \\
\text { Overlapping Figures Test. }\end{array}$ & - & - & - \\
\hline $\begin{array}{l}\text { Harrison } \\
\text { et al., } 2011 \\
{[26]}\end{array}$ & $\begin{array}{l}\mathrm{AN}=50 \\
(16-55) \\
\mathrm{BN}=48 \\
(16-55) \\
\text { AN- } \\
\mathrm{REC}=35 \\
(16-55) \\
\mathrm{HC}=89 \\
(16-55)\end{array}$ & $\begin{array}{l}\text { Fragmented } \\
\text { Pictures Task (CC) } \\
\text { Rey Complex } \\
\text { Figure Test (CC) } \\
\text { Group } \\
\text { Embedded } \\
\text { Figures Test (CC) }\end{array}$ & - & - & $\begin{array}{l}\text { Negative correlation } \\
\text { between BMI and } \\
\text { Fragmented Picture Task, } \\
\text { but not significant after } \\
\text { correcting for multiple } \\
\text { comparisons. }\end{array}$ & $\begin{array}{l}\text { Those with } \\
\text { reduced } \\
\text { central } \\
\text { coherence had } \\
\text { higher EDE-Q } \\
\text { Global score }\end{array}$ \\
\hline $\begin{array}{l}\text { McAnarney } \\
\text { et al., } 2011 \\
\text { [27] }\end{array}$ & $\begin{array}{l}\mathrm{AN}-\mathrm{R}= \\
24(14- \\
20) \\
\mathrm{HC}=37 \\
(14-20)\end{array}$ & $\begin{array}{l}\text { Wisconsin Card } \\
\text { Sorting Test (SS) } \\
\text { Intra/ } \\
\text { Extradimensional } \\
\text { Set Shift test (SS) }\end{array}$ & N/A & N/A & No significant correlations. & N/A \\
\hline $\begin{array}{l}\text { Bühren } \\
\text { et al., } 2012 \\
{[37]}\end{array}$ & $\begin{array}{l}\mathrm{AN}=28 \\
(15.6) \\
\mathrm{HC}=27 \\
(15.0)\end{array}$ & $\begin{array}{l}\text { Visual Set- } \\
\text { Shifting Task (SS) }\end{array}$ & - & - & N/A & $\mathrm{N} / \mathrm{A}$ \\
\hline $\begin{array}{l}\text { Danner } \\
\text { et al., } 2012 \\
\text { [38] }\end{array}$ & $\begin{array}{l}\mathrm{AN}=16 \\
(25.6) \\
\mathrm{AN}- \\
\mathrm{REC}=15 \\
(24.3) \\
\mathrm{HC}=15 \\
(25.8)\end{array}$ & $\begin{array}{l}\text { Berg's Card } \\
\text { Sorting Test (SS) } \\
\text { Rey Complex } \\
\text { Figure Test (CC) }\end{array}$ & $\begin{array}{l}\text { A better copy accuracy } \\
\text { on the Rey Complex } \\
\text { Figure Task was related } \\
\text { to higher levels of } \\
\text { anxiety. }\end{array}$ & - & N/A & - \\
\hline $\begin{array}{l}\text { Galimberti } \\
\text { et al., } 2012 \\
\text { [39] }\end{array}$ & $\begin{array}{l}\text { AN-R }= \\
24(26.7) \\
\text { AN-BP= } \\
12(27.1) \\
\text { BN }=16 \\
(25.3) \\
\text { HC }=40 \\
(25.9)\end{array}$ & $\begin{array}{l}\text { Intra/ } \\
\text { Extradimensional } \\
\text { Set shift test (SS) }\end{array}$ & - & - & - & $\mathrm{N} / \mathrm{A}$ \\
\hline $\begin{array}{l}\text { Giel et al., } \\
2012 \text { [40] }\end{array}$ & $\begin{array}{l}\mathrm{AN}=15 \\
(23.9) \\
\mathrm{UD}=20 \\
(36.3) \\
\mathrm{HC}=35 \\
(30.2)\end{array}$ & $\begin{array}{l}\text { Trail Making Test } \\
\text { (SS) } \\
\text { Wisconsin Card } \\
\text { Sorting Test (SS) } \\
\text { Parametric Go/ } \\
\text { No-Go Test (SS) }\end{array}$ & N/A & $\begin{array}{l}\text { Significant medium } \\
\text { correlations were found } \\
\text { in both AN and UD } \\
\text { patients. }\end{array}$ & - & - \\
\hline $\begin{array}{l}\text { Shott et al., } \\
2012 \text { [28] }\end{array}$ & $\begin{array}{l}\text { ADOL- } \\
\text { AN }=15 \\
(14.8) \\
\text { ADOL- } \\
C=16 \\
(14.0)\end{array}$ & $\begin{array}{l}\text { Category } \\
\text { Learning Task } \\
\text { (SS) }\end{array}$ & - & - & - & - \\
\hline
\end{tabular}


Table 1 Overview of associations between neuropsychological function and clinical measures in patients with AN. Significant associations are highlighted in bold (Continued)

\begin{tabular}{|c|c|c|c|c|c|c|}
\hline Authors & $\begin{array}{l}\text { Sample } \\
N \text { (mean } \\
\text { age) }\end{array}$ & Test & Anxiety & Depression & BMI/WfH & ED severity \\
\hline & $\begin{array}{l}\text { ADULT- } \\
\text { AN }=26 \\
(26.2) \\
\text { ADULT - } \\
C=33 \\
(26.0)\end{array}$ & & & & & \\
\hline $\begin{array}{l}\text { Galimberti } \\
\text { et al., } 2013 \\
{[41]}\end{array}$ & $\begin{array}{l}\mathrm{AN}=29 \\
(24.1) \\
\mathrm{HC}=29 \\
(28.6)\end{array}$ & $\begin{array}{l}\text { Wisconsin Card } \\
\text { Sorting Test (SS) }\end{array}$ & N/A & N/A & - & - \\
\hline $\begin{array}{l}\text { Tapajoz } \\
\text { et al., } 2013 \\
{[42]}\end{array}$ & $\begin{array}{l}\mathrm{AN}=24 \\
(24.5) \\
\mathrm{BN}=24 \\
(24.4) \\
\mathrm{HC}=24 \\
(25.2)\end{array}$ & $\begin{array}{l}\text { Rey Complex } \\
\text { Figure Task (CC) }\end{array}$ & - & - & - & - \\
\hline $\begin{array}{l}\text { Van } \\
\text { Autreve } \\
\text { et al., } 2013 \\
\text { [43] }\end{array}$ & $\begin{array}{l}\text { AN-R= } \\
31(26.0) \\
\text { AN-BP }= \\
20(20.0) \\
H C=26 \\
(19.0)\end{array}$ & $\begin{array}{l}\text { Block design (CC) } \\
\text { Object Assembly } \\
\text { (CC) } \\
\text { Wisconsin Card } \\
\text { Sorting Test (SS) } \\
\text { Trail Making Test } \\
\text { (SS) } \\
\text { Task Switching } \\
\text { Paradigm (SS) }\end{array}$ & $\begin{array}{l}\text { Trait anxiety in the AN-BP } \\
\text { group correlated with Ob- } \\
\text { ject Assembly. }\end{array}$ & - & - & N/A \\
\hline $\begin{array}{l}\text { Zuchova } \\
\text { et al., } 2013 \\
\text { [44] }\end{array}$ & $\begin{array}{l}\mathrm{AN}=31 \\
(25.6)\end{array}$ & $\begin{array}{l}\text { Rey Complex } \\
\text { Figure Task (CC) }\end{array}$ & N/A & N/A & - & $\begin{array}{l}\text { Significant } \\
\text { correlations } \\
\text { between recall } \\
\text { accuracy and } \\
\text { EDE-Q total } \\
\text { score and CR- } \\
\text { EAT }\end{array}$ \\
\hline $\begin{array}{l}\text { Abbate } \\
\text { Daga et al., } \\
2014 \text { [45] }\end{array}$ & $\begin{array}{l}\mathrm{AN}=94 \\
(24.7) \\
\mathrm{HC}=59 \\
(25.1)\end{array}$ & $\begin{array}{l}\text { Wisconsin Card } \\
\text { Sorting Test (SS) }\end{array}$ & N/A & $\begin{array}{l}\text { AN individuals with } \\
\text { poor set-shifting re- } \\
\text { ported higher BDI } \\
\text { scores than those with } \\
\text { intact set-shifting. }\end{array}$ & - & N/A \\
\hline $\begin{array}{l}\text { Aloi et al., } \\
2015 \text { [46] }\end{array}$ & $\begin{array}{l}\mathrm{AN}- \\
\mathrm{RES}=45 \\
(22.8) \\
\mathrm{BED}=45 \\
(30.6) \\
\mathrm{HC}=45 \\
(25.6)\end{array}$ & $\begin{array}{l}\text { Rey Complex } \\
\text { Figure Test (CC) } \\
\text { Trail Making Test } \\
\text { (SS) } \\
\text { Wisconsin Card } \\
\text { sorting Test (SS) }\end{array}$ & N/A & - & $\begin{array}{l}\text { BMI was associated with } \\
\text { poor cognitive flexibility, } \\
\text { extreme weight } \\
\text { conditions performed } \\
\text { worse than HC. }\end{array}$ & N/A \\
\hline $\begin{array}{l}\text { Lang et al., } \\
2015 \text { [22] }\end{array}$ & $\begin{array}{l}\mathrm{AN}=41 \\
(15.1) \\
\mathrm{HC}=43 \\
(15.1)\end{array}$ & $\begin{array}{l}\text { Wisconsin Card } \\
\text { Sorting Test (SS) } \\
\text { Rey Complex } \\
\text { Figure Test (CC) } \\
\text { Fragmented } \\
\text { Pictures Task (CC) }\end{array}$ & - & - & - & N/A \\
\hline $\begin{array}{l}\text { Renwick } \\
\text { et al., } 2015 \\
\text { [29] }\end{array}$ & $\begin{array}{l}\text { AN-R }= \\
44 \\
\text { AN-BP }= \\
33 \\
\text { EDNOS- } \\
\text { AN }=23 \\
\text { Total } \\
N=100 \\
(24.7)\end{array}$ & $\begin{array}{l}\text { Wisconsin Card } \\
\text { Sorting Test (SS) } \\
\text { Brixton Spatial } \\
\text { Anticipation Test } \\
\text { (SS) } \\
\text { Rey Complex } \\
\text { Figure Test (CC) }\end{array}$ & N/A & N/A & - & N/A \\
\hline
\end{tabular}


Table 1 Overview of associations between neuropsychological function and clinical measures in patients with AN. Significant associations are highlighted in bold (Continued)

\begin{tabular}{|c|c|c|c|c|c|c|}
\hline Authors & $\begin{array}{l}\text { Sample } \\
N \text { (mean } \\
\text { age) }\end{array}$ & Test & Anxiety & Depression & BMI/WfH & ED severity \\
\hline $\begin{array}{l}\text { Øverås } \\
\text { et al., } 2015 \\
\text { [47] }\end{array}$ & $\begin{array}{l}\text { AN-R= } \\
30(19.1) \\
H C=45 \\
(18.3)\end{array}$ & $\begin{array}{l}\text { Wisconsin Card } \\
\text { Sorting Test (SS) }\end{array}$ & $\begin{array}{l}\text { Significant correlations } \\
\text { with Wisconsin Card } \\
\text { Sorting Test. }\end{array}$ & $\begin{array}{l}\text { Significant correlations } \\
\text { with Wisconsin Card } \\
\text { Sorting Test. }\end{array}$ & $\begin{array}{l}\text { Significant correlations } \\
\text { with Wisconsin Card } \\
\text { Sorting Test. }\end{array}$ & N/A \\
\hline $\begin{array}{l}\text { Elzakkers } \\
\text { et al., } 2016 \\
\text { [48] }\end{array}$ & $\begin{array}{l}\mathrm{AN}=70 \\
(27.3)\end{array}$ & $\begin{array}{l}\text { Rey Complex } \\
\text { Figure Test (CC) } \\
\text { Wisconsin Card } \\
\text { sorting Test (SS) }\end{array}$ & N/A & N/A & - & N/A \\
\hline $\begin{array}{l}\text { Roberts } \\
\text { et al., } 2016 \\
\text { [49] }\end{array}$ & $\begin{array}{l}\mathrm{AN}=54 \\
(24.2)\end{array}$ & $\begin{array}{l}\text { Wisconsin Card } \\
\text { Sorting Test (SS) } \\
\text { Brixton Spatial } \\
\text { Anticipation Test } \\
\text { (SS) } \\
\text { Trail Making Test } \\
\text { (SS) } \\
\text { Haptic illusion } \\
\text { Task (SS) } \\
\text { Rey Complex } \\
\text { Figure Test (CC) } \\
\text { Group } \\
\text { Embedded } \\
\text { Figures Test (CC) }\end{array}$ & N/A & N/A & $\begin{array}{l}\text { The group with both } \\
\text { poor set-shifting and glo- } \\
\text { bal processing had a } \\
\text { lower lifetime BMI than } \\
\text { patients with one neuro- } \\
\text { cognitive inefficiency. }\end{array}$ & N/A \\
\hline $\begin{array}{l}\text { Rößner } \\
\text { et al., } 2016\end{array}$ & $\begin{array}{l}A N=69 \\
(14.3) \\
H C=63 \\
(14.1)\end{array}$ & $\begin{array}{l}\text { Trail Making Test } \\
\text { (SS) }\end{array}$ & N/A & - & - & N/A \\
\hline $\begin{array}{l}\text { Van Noort } \\
\text { et al., } 2016 \\
\text { [24] }\end{array}$ & $\begin{array}{l}\mathrm{EO}-\mathrm{AN}= \\
30(12.2) \\
\mathrm{AN}=30 \\
(15.9) \\
\mathrm{HC}=60 \\
(16.2)\end{array}$ & $\begin{array}{l}\text { Rey Complex } \\
\text { Figure Test (CC) } \\
\text { Trail Making Test } \\
\text { (SS) }\end{array}$ & N/A & N/A & - & N/A \\
\hline $\begin{array}{l}\text { Van Noort } \\
\text { et al., } 2016 \\
\text { [24] }\end{array}$ & $\begin{array}{l}\mathrm{AN}=20 \\
(15.6) \\
\mathrm{HC}=20 \\
(15.7)\end{array}$ & $\begin{array}{l}\text { Trail Making Test } \\
\text { (SS) } \\
\text { Rey Complex } \\
\text { Figure Test (CC) }\end{array}$ & N/A & N/A & - & N/A \\
\hline $\begin{array}{l}\text { Weider } \\
\text { et al., } 2016 \\
\text { [50] }\end{array}$ & $\begin{array}{l}\mathrm{AN}=41 \\
(28.2) \\
\mathrm{BN}=40 \\
(27.7) \\
\mathrm{HC}=41 \\
(27.7)\end{array}$ & $\begin{array}{l}\text { Rey Complex } \\
\text { Figure Test (CC) }\end{array}$ & N/A & N/A & $\begin{array}{l}\text { Significant low central } \\
\text { coherence index became } \\
\text { non-significant when } \\
\text { adjusting for nadir BMI. }\end{array}$ & N/A \\
\hline $\begin{array}{l}\text { Bentz et al., } \\
2017 \text { [25] }\end{array}$ & $\begin{array}{l}\text { FeAN = } \\
43(16.1) \\
\text { AN- } \\
\text { REC }=28 \\
(18.4) \\
H C=41 \\
(17.7)\end{array}$ & $\begin{array}{l}\text { Verbal fluency } \\
\text { Task (SS) } \\
\text { Design fluency } \\
\text { Task (SS) } \\
\text { Trail Making Test } \\
\text { (SS) } \\
\text { Group } \\
\text { Embedded } \\
\text { Figures Test (CC) }\end{array}$ & - & - & N/A & N/A \\
\hline $\begin{array}{l}\text { Perpiña } \\
\text { et al., } 2016 \\
{[51]}\end{array}$ & $\begin{array}{l}\text { AN-R }= \\
18(22.3) \\
\text { ED.AN= } \\
21(23.1) \\
\text { BP-G }= \\
47(24.5) \\
\mathrm{OB}=27 \\
(47.8) \\
\mathrm{HC}=39 \\
(31.9)\end{array}$ & $\begin{array}{l}\text { Wisconsin Card } \\
\text { Sorting Test (SS) }\end{array}$ & $\begin{array}{l}\text { Positive relationship } \\
\text { between anxiety and } \\
\text { "total errors" on } \\
\text { Wisconsin Card sorting } \\
\text { Test. Negative } \\
\text { relationship between } \\
\text { anxiety and "total } \\
\text { categories completed" on } \\
\text { the Wisconsin Card } \\
\text { Sorting Test. }\end{array}$ & $\begin{array}{l}\text { A positive relationship } \\
\text { between depression } \\
\text { and "total errors" on } \\
\text { Wisconsin card sorting } \\
\text { test, and negative } \\
\text { correlations between } \\
\text { depression and lowa } \\
\text { gambling task in all } \\
\text { groups. }\end{array}$ & N/A & - \\
\hline
\end{tabular}


Table 1 Overview of associations between neuropsychological function and clinical measures in patients with AN. Significant associations are highlighted in bold (Continued)

\begin{tabular}{|c|c|c|c|c|c|c|}
\hline Authors & $\begin{array}{l}\text { Sample } \\
N \text { (mean } \\
\text { age) }\end{array}$ & Test & Anxiety & Depression & BMI/WfH & ED severity \\
\hline $\begin{array}{l}\text { Øverås } \\
\text { et al., } 2017 \\
\text { [52] }\end{array}$ & $\begin{array}{l}\mathrm{AN}=35 \\
(18.8) \\
\mathrm{HC}=34 \\
(18.7)\end{array}$ & $\begin{array}{l}\text { Rey Complex } \\
\text { Figure Test (CC) } \\
\text { Wisconsin Card } \\
\text { Sorting Test (SS) }\end{array}$ & - & - & - & N/A \\
\hline $\begin{array}{l}\text { Brown } \\
\text { et al., } 2018 \\
\text { [18] }\end{array}$ & $\begin{array}{l}\mathrm{AN}=10 \\
(14.5) \\
\mathrm{HC}=14 \\
(13.7) \\
\mathrm{AN} \\
\text { parent }= \\
10(44.7) \\
\mathrm{HC} \\
\text { parent }= \\
14(46.9)\end{array}$ & $\begin{array}{l}\text { Rey Complex } \\
\text { Figure Test (CC) } \\
\text { Group } \\
\text { Embedded } \\
\text { Figure Test (CC) } \\
\text { Trail Making Test } \\
\text { (SS) } \\
\text { Verbal Fluency } \\
\text { Test (SS) }\end{array}$ & $\begin{array}{l}\text { Higher levels of anxiety } \\
\text { could affect cognitive } \\
\text { function. }\end{array}$ & $\begin{array}{l}\text { Higher levels of } \\
\text { depression could affect } \\
\text { cognitive function. }\end{array}$ & N/A & N/A \\
\hline $\begin{array}{l}\text { Hamatani } \\
\text { et al., } 2018 \\
\text { [53] }\end{array}$ & $\begin{array}{l}\mathrm{AN}=22 \\
(31.5) \\
\mathrm{HC}=33 \\
(28.0)\end{array}$ & $\begin{array}{l}\text { Rey Complex } \\
\text { Figure Test (CC) }\end{array}$ & - & - & - & N/A \\
\hline $\begin{array}{l}\text { Herbrich } \\
\text { et al., } 2018 \\
\text { [21] }\end{array}$ & $\begin{array}{l}\text { AN-R }= \\
90(14.4) \\
\text { AN-BP }= \\
21(15.2) \\
H C=63 \\
(14.0)\end{array}$ & $\begin{array}{l}\text { Trail Making Test } \\
\text { (SS) } \\
\text { Group } \\
\text { Embedded } \\
\text { Figures Test (CC) } \\
\text { Rey Complex } \\
\text { Figure Test (CC) } \\
\text { Wisconsin Card } \\
\text { Sorting Test (SS) }\end{array}$ & - & - & $\begin{array}{l}\text { Negative correlation } \\
\text { between BMI and Trail } \\
\text { Making Test- } 4 \text { in the AN- } \\
\text { R group. Negative correl- } \\
\text { ation between BMI and } \\
\text { Group Embedded Figures } \\
\text { Test in the AN-BP group. }\end{array}$ & N/A \\
\hline $\begin{array}{l}\text { Leppanen } \\
\text { et al., } 2018 \\
\text { [54] }\end{array}$ & $\begin{array}{l}\mathrm{AN}=145 \\
(25.0)\end{array}$ & $\begin{array}{l}\text { Rey Complex } \\
\text { Figure Test (SS) } \\
\text { Brixton Spatial } \\
\text { Anticipation Test } \\
\text { (SS) }\end{array}$ & N/A & N/A & - & N/A \\
\hline $\begin{array}{l}\text { Oldershaw } \\
\text { et al., } 2018 \\
\text { [55] }\end{array}$ & $\begin{array}{l}A N=71 \\
(26.6)\end{array}$ & $\begin{array}{l}\text { Wisconsin Card } \\
\text { Sorting Test (SS) } \\
\text { Brixton Spatial } \\
\text { Anticipation test } \\
\text { (SS) } \\
\text { Trail Making Test } \\
\text { (SS) } \\
\text { Group } \\
\text { Embedded } \\
\text { Figure Test (CC) }\end{array}$ & N/A & N/A & - & N/A \\
\hline $\begin{array}{l}\text { Berner et al. } \\
2019 \text { [56] }\end{array}$ & $\begin{array}{l}\text { IAN }=40 \\
(24.3) \\
\text { RAN }=24 \\
(25.6) \\
\text { HC }=42 \\
(22.0)\end{array}$ & $\begin{array}{l}\text { Cued Color- } \\
\text { Shape Switching } \\
\text { Task (SS) }\end{array}$ & - & - & - & N/A \\
\hline $\begin{array}{l}\text { Sproch } \\
\text { et al. } 2019 \\
\text { [57] }\end{array}$ & $\begin{array}{l}\text { AN } \\
\text { CRT }= \\
135 \\
(23.9) \\
\text { AN CC= } \\
140 \\
(22.2)\end{array}$ & $\begin{array}{l}\text { Wisconsin Card } \\
\text { Sorting Test (SS) } \\
\text { Comprehensive } \\
\text { Trail Making Test } \\
\text { (SS) }\end{array}$ & N/A & N/A & - & N/A \\
\hline
\end{tabular}

$A D O L-A N$ adolescent patients with $\mathrm{AN}, A D O L-C$ adolescent controls, $A D U L T-A N$ adult patients with AN, $A D U L T-C$ adult control women, $A N$ anorexia nervosa, $A N-B P$ anorexia nervosa binge/purge subtype, $A N C C$ anorexia nervosa control condition, $A N C R T$ anorexia nervosa cognitive remediation therapy, $A N-R$ anorexia nervosa restrictive subtype, $A N-R E S$ anorexia nervosa restrictive subtype, $A N-R E C$ anorexia nervosa recovered, $B D I$ Beck's Depression Inventory, $B E D$ binge eating disorder, $B N$ bulimia nervosa, $C C$ Central Coherence, $E D$ eating disorder, $E D . A N$ eating disorder not otherwise specified anorexia nervosa type, $E O-A N$ early onset anorexia nervosa, FeAN first-episode AN; HC, healthy controls, IAN ill with AN, subthreshold AN or atypical AN, N/A not applicable, BP-G binge/purge group, OB obese group, RAN remitted from AN, SS set shifting, UD patients with unipolar depression, WfH weight for height ratio.s 
including measures of set-shifting show that BMI was significantly associated with poor cognitive flexibility, were extreme weight conditions performed worse than healthy controls [46]. Øverås et al. demonstrated significant correlations between BMI and Wisconsin Card Sorting Test [47], and Roberts et al. [49] reported that the group with poor set-shifting abilities and global processing had a lower lifetime BMI than patients with only one neurocognitive inefficiency.

\section{Anxiety and neuropsychological function}

Most studies $(n=16)$ did not find an association between neuropsychological function and anxiety [5, 19$22,25,26,28,30,32,34,39,42,52,53,56,59]$, while nine studies did find a significant association. One study showed that group differences in a Cognitive Shift Task disappeared when controlling for anxiety [59]. Another study found that anxiety scored correlated with the Haptic Illusion task, a measure of set-shifting [31]. Roberts et al. [35] found increased anxiety in the poor setshifting group, however the effects were small. Another study showed that state anxiety had a moderate correlation with Trail Making Test and Overlapping Figures Test [36]. Øverås et al. and Perpiña et al. also found that set-shifting correlated with anxiety [47, 51]. A better copy accuracy on the Rey Complex Figure Task was related to higher levels of anxiety [38], while trait anxiety in the AN-BP group correlated with Object Assembly, a measure of central coherence. Brown and colleagues [18] suggest that higher levels of anxiety could affect cognitive function.

\section{Depression and neuropsychological function}

A total of 20 studies reported that depression was not associated with neuropsychological function $[5,19-23$, $25,26,28,30,33,36,38,39,42,43,46,52,53,56,59]$. However, nine studies did find significant correlations between depression and measures of set-shifting and central coherence. These studies demonstrate correlations between depression and the Haptic Illusion Task [31], and set-shifting measured by Trail Making Test [34]. Another study found increased depression in the poor set-shifting group, however with small effects [35]. Medium correlations between set-shifting and depression was found in both patients with $\mathrm{AN}$ and patients with unipolar depression [40]. Abbate Daga et al. [45] found that AN individuals with poor set-shifting reported higher depression scores, measured by Beck's Depression Inventory, than those patients intact setshifting. Furthermore, studies by Øverås et al. [47] and Perpiña et al. [51] show significant correlations between depression and Wisconsin Card Sorting Test. Brown et al. [18] also suggest that higher levels of depression could affect cognitive function such as central coherence and set-shifting.

\section{Eating disorder severity and neuropsychological function} Some studies investigated the associations between eating disorder severity and neuropsychological functions. Individuals with poor set-shifting had a higher score on the Yale-Brown-Cornell Eating Disorder Scale rituals [35]. Those with most fragmented perseverative cognitive style and the least global flexible style had more chronic and more severe illness, in terms of higher Eating disorder examination questionnaire (EDE-Q) global score and lower BMI [26]. Significant correlations were found between recall accuracy on the Rey Complex Figure Test and total EDE-Q score and scores on the Clinical and Research Inventory for Eating Disorders. In addition, an increase in eating disorder symptoms was related to an improvement in set-shifting measured by the trail making test/TMT [44].

Conversely, other studies showed no correlations between set-shifting performance $[32,40]$ or central coherence measured by the Rey Complex Figure Task [42] on scores on the Eating Disorder Inventory. Another study found no evidence that symptoms of eating disorders, as measured by EDE-Q, was associated with set-shifting ability [34]. Tenconi et al. [36] divided individuals with AN into three groups, acute AN, weight-recovered but symptomatic AN and long-term fully recovered AN. There were no differences between the groups on measures of set-shifting and central coherence. Furthermore, Danner et al. [38] found no correlations between Iowa Gambling Task and clinical characteristics, while Shott et al. [28] found no significant correlations between shift-cost index on the Category Learning Task and duration of illness. In another study, severity, assessed by the Yale-Brown-Cornell scale scores, BMI, onset and duration of illness, did not correlate with any neurocognitive performance [41]. One study found no significant associations between the Eating Attitudes Test scores and the Iowa Gambling Task total net scores or the Wisconsin Card Sorting Test [51].

\section{Discussion}

The aim of this study was to systematically review the literature to evaluate whether the observed alterations in set-shifting and central coherence are related to BMI or transdiagnostical factors such as anxiety and depression. The JBI Critical Appraisal has shown that the majority studies in this review had a low risk of bias. However, some studies were uncertain of the representativeness of their experimental group, or did not appropriately control for confounding factors such as intelligence quotient, pharmacological treatment or comorbidity, leading to a modest increase in risk of bias in these studies. 
Main findings, the role of neuropsychological functions in $\mathrm{AN}$ and clinical implications will be further discussed.

\section{Neuropsychological function and clinical measures}

The majority of studies report that neither BMI, eating disorder severity, anxiety nor depression is associated with neuropsychological task performance. Deficits in set-shifting and central coherence could thus be related to characteristics of the disorder, and are not a direct consequence of underweight, ED severity, anxiety or depression. However, although most findings points to the same direction, findings are inconsistent. The studies are heterogeneous and methodological differences needs to be considered. Various tests have been used to measure set-shifting and central coherence, one might therefore question whether these results are comparable, as different cognitive tests have different levels of complexity and might target multiple cognitive capabilities. For example, the WCST is primarily a measure of cognitive flexibility, however other cognitive functions such as working memory, inhibition and sustained attention are also necessary to perform this task. The Embedded Figures test appears to the participants to be a measure of attention, but is rather a visuospatial perceptual task measuring central coherence ability. A standardized approach to neuropsychological testing of individuals with AN could contribute to increased consistency across studies, and a better understanding of the neuropathology. Another important issue is that there are numerous measures of psychopathology, and especially for anxiety and depression. Even if they are supposed to measure the same constructs, results from different instruments can be difficult to compare [60].

Furthermore, different statistical methods have been used to determine the relationship between neuropsychological function and clinical measures. Most studies have used correlation analyses (i.e. Pearson or Spearman's), however some studies did multivariate regression analyses including one or more covariates. The different methods could lead to different conclusions, as a correlation analysis does not adjust for the effect of other covariates.

Neuropsychological performance could also depend on medical treatment. A meta-analysis suggest that deficits in executive functions are greater in patients with more severe current depression, and those taking psychotropic medications [61]. In this review, a few studies accounted for medical treatment and whether it could influence neuropsychological function. Most of these studies suggested that medication did not influence test performance $[26,28,31,34,37]$, while two studies found differences between medicated and non-medicated patients [36, 42]. Some studies had long-term use of medication $[23,24,58]$ and current treatment with psychotropic drugs [25] as exclusion criteria.

It is important to note that cognitive development most likely impact neuropsychological function. Most neuropsychological studies have an adult sample, and studies with an adolescent AN sample are inconsistent, showing both weak and normal set-shifting $[27,62,63]$ normal central coherence [21] as well as lower scores on style and central coherence index measured by Rey Complex Figure Test [22]. One study directly compared set-shifting performance in adolescents versus adults with AN and controls [28]. The results showed that set-shifting is normal in adolescents with AN, but impaired in adults, suggesting that neuropsychological difficulties do not precede the disorder, however are rather accompanied by the eating disorder symptoms. Whether neuropsychological function is influenced by clinical measures in adolescents remains unclear. Some studies showed a negative correlation between BMI and both setshifting and central coherence [21], and that both anxiety and depression could affect cognitive function [18]. Conversely, quite a few studies did not find any significant relationship between set-shifting or central coherence and BMI, anxiety and depression [19, 22-24, 28].

\section{Neuropsychological function as a link between underlying neurobiological alterations and AN symptoms and behaviour}

The main purpose of cognitive neuroscience research in AN is to explain the link between symptoms/behaviour and underlying neural systems. Abnormalities in the fronto-striatal and limbic systems have been suggested to be involved in restrictive eating, which is one of the core symptoms of AN [64]. Central coherence and setshifting are both executive functions, which rely on frontal lobe activity. More specifically, during a setshifting task, patients with $\mathrm{AN}$ have shown activation in ventrolateral prefrontal cortex, dorsolateral prefrontal cortex, anterior cingulate, superior parietal cortex, cerebellum and precuneus. When performing a central coherence task, parietal, occipital, ventral temporal and premotor cortex is usually activated [65]. Maladaptive behaviours are associated with specific traits, which in turn are associated with neuropsychological processes that are related to specific neural circuits [66]. Clinically, impairments in set-shifting in AN could be linked to excessive control of eating and weight, but also cognitive and behavioural rigidity, which is a common characteristic of the disorder. Patients with AN are often "stuck" in maladaptive behaviours, and have difficulties to change these behavioural patterns. Cognitive and behavioural rigidity could be expressed in terms of being unable to shift perspective from detailed rules and rituals concerning food and eating [6]. Another typical characteristic of individuals with $\mathrm{AN}$, is the increased attention to details, 
which could be linked to poor global processing. They often have detailed rituals concerning food, caloric intake, exercise and body checking. Taken together, inflexibility and an excessive control of details concerning food and weight, could contribute to the unusual ability to reduce weight and sustain an extreme low weight over a long period.

AN can be very difficult to treat, and there is no proven treatment that can reverse the core symptoms. A complete understanding of neuropsychological functioning in $\mathrm{AN}$, as a link between neural mechanisms and AN symptoms, could contribute to the development of better treatment strategies. As suggested in this review, neuropsychological functions are not influenced by low weight or comorbid disorders, but might be a trait of the illness and should be targeted in treatment with that in mind.

As a result of the emerging neurobiological models of $\mathrm{AN}$, it has been suggested that there is a need for a paradigm shift in the development of new treatment strategies, with more focus on brain-directed treatments [67]. Findings from neuroscientific research has opened up for new avenues for understanding the underlying mechanisms and neuropsychological functions for AN, and for developing new treatments. Temperament based treatment with support (TBT-S) [68] is based on models explaining the underlying neurobiological mechanisms that cause AN. The goal of this treatment is to teach individuals with AN to develop coping strategies for managing their temperament traits that are related to risk factors to develop an eating disorder. The treatment is delivered in a way that is compatible with their neuropsychological thinking style, i.e. detailed oriented, wellstructured and predictable. The Maudsley Model of Anorexia Nervosa Treatment for Adults (MANTRA), aims to address the neuropsychological, emotional, relational and biological aspects of AN. It is based on research that has identified the key factors that tend to maintain eating difficulties [69]. The treatment helps patients to understand what causes AN, as well as to encourage patients to change their behaviour when they are ready for it. Cognitive remediation therapy (CRT) aims at improving cognitive flexibility and central coherence, as well as reducing perfectionism and improving the awareness of dysfunctional thinking styles. This treatment is focusing on the process rather than the content of thinking [70].

\section{Limitations and future directions}

Neuropsychological research in AN is distinguished by large varieties of tasks within the same cognitive domain and a variety of measures of psychopathology, which impact and limit interpretation of findings and comparison of results across studies. In a systematic review, a quantitative synthesis is considered to be superior methodologically compared to a narrative synthesis of the results.
However, due to the heterogeneity of the primary studies, especially considering the wide variety of neuropsychological tests and measures of psychopathology and that different statistical methods have been used, a narrative synthesis approach was used. Another limitation with the existing literature is that few studies include an adolescent sample, who have a short duration of illness, and addressing this group could contribute to an increased understanding of predisposing factors of AN. Additional issues still needs to be addressed, such as when do the neuropsychological impairments occur? Are neuropsychological impairments related to age or to the progression of the disorder? Do neuropsychological impairments contribute to maintain the disorder? Future studies should target these issues, which could contribute to an increased understanding of the neuropathology of AN.

Future studies would also benefit from controlling for the potential effect of medical treatment on neuropsychological performance, include associations between neuropsychological functioning and eating disorder severity, by including other relevant measures that might have clinical relevance in addition to BMI, e.g. EDE-Q, or other measures of eating disorder symptoms.

\section{Conclusions}

Although the results are inconsistent, the majority of studies in this review report that BMI, anxiety and depression do not influence set-shifting and central coherence capacities in AN. This could indicate that setshifting and central coherence are characteristic of the disorder per se, and not a consequence of comorbid psychopathologies or emaciation. However, which factors that contribute to altered neuropsychological function in AN are yet to be established. A complete understanding of predisposing, precipitating and maintaining factors of AN needs to be addressed in future research. This could contribute to the development of better and more targeted treatment strategies.

\section{Abbreviations}

AN: Anorexia nervosa; BMI: Body mass index; CRT: Cognitive remediation therapy; EDE-Q: Eating disorder examination questionnaire; MANTRA: The Maudsley Model of Anorexia Nervosa Treatment for Adults; TBT-

S: Temperament based treatment with support

\section{Supplementary Information}

The online version contains supplementary material available at https://doi. org/10.1186/s12888-021-03120-6.

Additional file 1. Search strategy in Pubmed. Illustrates the search strategy that was used in PubMed

Additional file 2. Checklist_for_Analytical_Cross_Sectional_Studies Critical appraisal tool from the Joanna Briggs Institute, for cross sectional studies included in systematic reviews. 
Additional file 3. Checklist for Case_Control_Studies. Critical appraisa tool from the Joanna Briggs Institute, for case control studies included in systematic reviews.

\section{Acknowledgements}

The author would like to thank Trine Sandblost and Eva Margrethe Lundanes for contributing to the literature search.

\section{Availability of data and material}

No data or materials were generated for this systematic review, only summarized data is presented in the manuscript.

\section{Author's contributions}

TSF conducted the literature search, analyzed the data and wrote the paper. The authors read and approved the final manuscript.

\section{Authors' information}

TSF has a PhD in Psychology from the University of Oslo and is currently in a postdoctoral position in Møre and Romsdal Hospital Trust in Molde, Norway.

\section{Funding}

The author of this study is currently in a postdoctoral position funded by Møre and Romsdal Hospital Trust in Norway. The design of the study, collection, analysis, interpretation of data and writing of the manuscript was funded by Møre and Romsdal Hospital Trust.

\section{Ethics approval and consent to participate}

Not applicable.

\section{Consent for publication}

Not applicable.

\section{Competing interests}

The author declare that there are no competing interests.

\section{Received: 28 February 2020 Accepted: 17 February 2021} Published online: 08 March 2021

\section{References}

1. American Psychiatric Association. Diagnostic and Statistical Manual of Mental Disorders: Diagnostic and Statistical Manual of Mental Disorders, Fifth Edition. Arlington: American Psychiatric Association; 2013.

2. Arcelus J, Mitchell AJ, et al. Mortality rates in patients with anorexia nervosa and other eating disorders. A meta-analysis of 36 studies. Arch Gen Psychiatry. 2011;68(7):724-31.

3. Watson HJ, Yilmaz Z, et al. Genome-wide association study identifies eight risk loci and implicates metabo-psychiatric origins for anorexia nervosa. Nat Genet. 2019:51(8):1207-14

4. Lang K, Lopez C, et al. Central coherence in eating disorders: an updated systematic review and meta-analysis. World J Biol Psychiatr. 2014:15(8):586-98

5. Lopez C, Tchanturia K, et al. An examination of the concept of central coherence in women with anorexia nervosa. Int J Eat Disord. 2008:41(2): $143-52$

6. Smith $\mathrm{KE}$, Mason TB, et al. A systematic review of reviews of neurocognitive functioning in eating disorders: the state-of-the-literature and future directions. Int J Eat Disord. 2018:51 (8):798-821.

7. Lang K, Stahl D, et al. Set shifting in children and adolescents with anorexia nervosa: an exploratory systematic review and meta-analysis. Int J Eat Disord. 2014:47:394-9.

8. Lang K. Tchanturia K. A systematic Review of Central Coherence in Young People with Anorexia Nervosa. J Child Adolesc Behav. 2014:2:140

9. Blakemore SJ, Choudhury S. Development of the adolescent brain: implications for executive function and social cognition. J Child Psychol Psychiatry. 2006;47(3-4):296-312.

10. Ranseen JD, Humphries LL. The intellectual functioning of eating disorder patients. J Am Acad Child Adolesc Psychiatry. 1992;31(5):844-6.

11. Southgate $L$, Tchanturia $K$, et al. Information processing bias in anorexia nervosa. Psychiatry Res. 2008;160(2):221-7.
12. Roberts ME, Tchanturia $\mathrm{K}$, et al. Is attention to detail a similarly strong candidate endophenotype for anorexia nervosa and bulimia nervosa? World J Biol Psychiatr. 2013;14(6):452-63.

13. Lopez C, Stahl D, et al. Estimated intelligence quotient in anorexia nervosa: a systematic review and meta-analysis of the literature. Ann General Psychiatry. 2010:9:40.

14. Marucci S, Ragione LD, et al. Anorexia nervosa and comorbid psychopathology. Endocr Metab Immune Disord Drug Targets. 2018;18(4): $316-24$

15. Burt DB, Zembar MJ, et al. Depression and memory impairment: a metaanalysis of the association, its pattern, and specificity. Psychol Bull. 1995; 117(2):285-305.

16. Castaneda $A E$, Tuulio-Henriksson A, et al. A review on cognitive impairments in depressive and anxiety disorders with a focus on young adults. J Affect Disord. 2008:106(1-2):1-27.

17. Moher $D$, Shamseer $L$, et al. Preferred reporting items for systematic review and meta-analysis protocols (PRISMA-P) 2015 statement. Syst Rev. 2015:4(1):1

18. Brown M, Loeb $\mathrm{KL}$, et al. Executive functioning and central coherence in anorexia nervosa: pilot investigation of a neurocognitive endophenotype. Eur Eat Disord Rev. 2018;26(5):489-98.

19. Buhren K, Mainz V, et al. Cognitive flexibility in juvenile anorexia nervosa patients before and after weight recovery. J Neural Trans (Vienna, Austria : 1996). 2012;119(9):1047-57.

20. Fowler $L$, Blackwell A, et al. Profile of neurocognitive impairments associated with female in-patients with anorexia nervosa. Psychol Med. 2006;36(4):517-27.

21. Herbrich L, Kappel $V$, et al. Differences in set-shifting and central coherence across anorexia nervosa subtypes in children and adolescents. Eur Eat Disord Rev. 2018:26(5):499-507.

22. Lang K, Lloyd S, et al. Do children and adolescents with anorexia nervosa display an inefficient cognitive processing style? PLoS One. 2015:10(7): e0131724.

23. Rossner A, Juniak I, van Noort BM, Pfeiffer E, Lehmkuhl U, Kappel V. Cognitive flexibility in juvenile anorexia Nervosain relation to comorbid symptoms of depression, obsessive compulsive symptoms and duration of illness. Zeitschrift Kinder. 2017;45(5):371-80.

24. van Noort BM, Pfeiffer $E$, et al. Cognitive performance in children with acute early-onset anorexia nervosa. Eur Child Adolesc Psychiatry. 2016;25(11): 1233-44.

25. Bentz $M$, Jepsen JRM, et al. Neurocognitive functions and social functioning in young females with recent-onset anorexia nervosa and recovered individuals. J Eat Disord. 2017;5:5.

26. Harrison A, Tchanturia K, et al. Measuring state trait properties of detail processing and global integration ability in eating disorders. World J Biol Psychiatr. 2011;12(6):462-72.

27. McAnarney ER, Zarcone J, et al. Restrictive anorexia nervosa and setshifting in adolescents: a biobehavioral interface. J Adolesc Health. 2011:49(1):99-101.

28. Shott ME, Filoteo JV, et al. Cognitive set-shifting in anorexia nervosa. Eur Eat Disord Rev. 2012:20(5):343-9.

29. Renwick B, Musiat P, et al. Neuro- and social-cognitive clustering highlights distinct profiles in adults with anorexia nervosa. Int J Eat Disord. 2015:48(1): 26-34.

30. Tchanturia K, Morris RG, et al. Set shifting in anorexia nervosa: an examination before and after weight gain, in full recovery and relationship to childhood and adult OCPD traits. J Psychiatr Res. 2004:38(5):545-52.

31. Holliday J, Tchanturia $K$, et al. Is impaired set-shifting an endophenotype of anorexia nervosa? Am J Psychiatry. 2005;162(12):2269-75.

32. Steinglass J, Walsh BT. Habit learning and anorexia nervosa: a cognitive neuroscience hypothesis. Int J Eat Disord. 2006;39(4):267-75.

33. Steinglass JE, Walsh BT, et al. Set shifting deficit in anorexia nervosa. J Int Neuropsychol Soc. 2006;12(3):431-5.

34. Kim YR, Kim JE, et al. Impaired set-shifting ability in patients with eating disorders, which is not moderated by their catechol-O-methyltransferase Val158Met genotype. Psychiatry Investig. 2010;7(4):298-301.

35. Roberts ME, Tchanturia $\mathrm{K}$, et al. Exploring the neurocognitive signature of poor set-shifting in anorexia and bulimia nervosa. J Psychiatr Res. 2010; 44(14):964-70.

36. Tenconi $E$, Santonastaso $P$, et al. Set-shifting abilities, central coherence, and handedness in anorexia nervosa patients, their unaffected siblings and healthy controls: exploring putative endophenotypes. World J Biol Psychiatr. 2010;11(6):813-23. 
37. Bühren $K$, Schwarte $R$, et al. Comorbid psychiatric disorders in female adolescents with first-onset anorexia nervosa. Eur Eat Disord Rev. 2014;22(1): 39-44.

38. Danner UN, Sanders N, et al. Neuropsychological weaknesses in anorexia nervosa: set-shifting, central coherence, and decision making in currently ill and recovered women. Int J Eat Disord. 2012;45(5):685-94.

39. Galimberti E, Martoni RM, et al. Motor inhibition and cognitive flexibility in eating disorder subtypes. Prog Neuro-Psychopharmacol Biol Psychiatry. 2012;36(2):307-12.

40. Giel KE, Wittorf A, et al. Is impaired set-shifting a feature of "pure" anorexia nervosa? Investigating the role of depression in set-shifting ability in anorexia nervosa and unipolar depression. Psychiatry Res. 2012;200(2-3): 538-43.

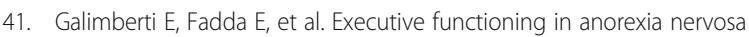
patients and their unaffected relatives. Psychiatry Res. 2013;208(3):238-44.

42. Tapajoz PSF, Soneira S, et al. Theory of mind and central coherence in eating disorders: two sides of the same coin? Psychiatry Res. 2013;210(3): $1116-22$.

43. Van Autreve S, De Baene W, et al. Do restrictive and bingeing/purging subtypes of anorexia nervosa differ on central coherence and set shifting? Eur Eat Disord Rev. 2013;21(4):308-14.

44. Zuchova S, Kubena AA, et al. Neuropsychological variables and clinical status in anorexia nervosa: relationship between visuospatial memory and central coherence and eating disorder symptom severity. Eat Weight Disord. 2013;18(4):421-8.

45. Abbate-Daga G, Buzzichelli S, Marzola E, Amianto F, Fassino S. Clinical investigation of set-shifting subtypes in anorexia nervosa. Psychiatry Res. 2014;219(3):592-7.

46. Aloi M, Rania M, et al. Decision making, central coherence and set-shifting: a comparison between binge eating disorder, Anorexia Nervosa and Healthy Controls. BMC Psychiatr. 2015;15:6.

47. Overas M, Kapstad H, Brunborg C, Landro NI, Lask B. Are poor set-shifting abilities associated with a higher frequency of body checking in anorexia nervosa? J Eat Disord. 2015;3:17.

48. Elzakkers IF, Danner UN, et al. Mental capacity to consent to treatment in anorexia nervosa: explorative study. BJPsych Open. 2016;2(2):147-53.

49. Roberts ME, Tchanturia $K$, et al. Overlapping neurocognitive inefficiencies in anorexia nervosa: a preliminary investigation of women with both poor setshifting and weak central coherence. Eat Weight Disord. 2016;21(4):725-9.

50. Weider S, Indredavik MS, et al. Central coherence, Visuoconstruction and visual memory in patients with eating disorders as measured by different scoring methods of the Rey complex figure test. Eur Eat Disord Rev. 2016; 24(2):106-13.

51. Perpina C, Segura M, et al. Cognitive flexibility and decision-making in eating disorders and obesity. Eating and weight disorders: EWD. 2016.

52. Overas M, Kapstad H, Brunborg C, Landro NI, Rø Ø. Is overestimation of body size associated with neuropsychological weaknesses in anorexia nervosa? Eur Eat Disord Rev. 2017;25(2):129-34.

53. Hamatani S, Tomotake M, Takeda T, Kameoka N, Kawabata M, Kubo H, et al. Impaired central coherence in patients with anorexia nervosa. Psychiatry Res. 2018;259:77-80.

54. Leppanen J, Adamson J, et al. Impact of cognitive remediation therapy on neurocognitive processing in anorexia nervosa. Front Psychiatr. 2018;9:96.

55. Oldershaw A, Lavender T, et al. Are socio-emotional and neurocognitive functioning predictors of therapeutic outcomes for adults with anorexia nervosa? Eur Eat Disord Rev. 2018;26(4):346-59.

56. Berner LA, Romero EM, et al. Task-switching inefficiencies in currently ill, but not remitted anorexia nervosa. Int J Eat Disord. 2019;52(11):1316-21.

57. Sproch LE, Anderson KP, et al. A randomized controlled trial of group cognitive remediation therapy for anorexia nervosa: effects on set-shifting tasks for inpatient adults and adolescents. Int J Eat Disord. 2019;52(9):1004-14.

58. van Noort BM, Kraus MK, et al. Neuropsychological and Behavioural shortterm effects of cognitive remediation therapy in adolescent anorexia nervosa: a pilot study. Eur Eat Disord Rev. 2016;24(1):69-74.

59. Tchanturia K, Morris RG, et al. An examination of perceptual and cognitive set shifting tasks in acute anorexia nervosa and following recovery. Eat Weight Disord. 2002;7(4):312-5

60. Wahl I, Löwe B, et al. Standardization of depression measurement: a common metric was developed for 11 self-report depression measures. J Clin Epidemiol. 2014;67(1):73-86.
61. Snyder HR. Major depressive disorder is associated with broad impairments on neuropsychological measures of executive function: a meta-analysis and review. Psychol Bull. 2013;139(1):81-132.

62. Dmitrzak-Weglarz M, Slopien A, et al. Polymorphisms of the SNAP-25 gene and performance on the Wisconsin card sorting test in anorexia nervosa and in healthy adolescent participants. Arch Psychiatry Psychother. 2011;1: 43-51.

63. Sarrar L, Ehrlich $\mathrm{S}$, et al. Cognitive flexibility and agouti-related protein in adolescent patients with anorexia nervosa. Psychoneuroendocrinology. 2011;36(9):1396-406

64. Kaye $W H$, Fudge $J$, et al. New insights into symptoms and neurocircuit function of anorexia nervosa. Nat Rev Neurosci. 2009;10(8):573-84.

65. Garrett AS, Lock J, et al. Predicting clinical outcome using brain activation associated with set-shifting and central coherence skills in anorexia nervosa. J Psychiatr Res. 2014;57:26-33.

66. Steinglass JE, Walsh BT. Neurobiological model of the persistence of anorexia nervosa. J Eat Disord. 2016:4:19.

67. Schmidt U, Campbell IC. Treatment of eating disorders can not remain 'brainless': the case for brain-directed treatments. Eur Eat Disord Rev. 2013; 21(6):425-7.

68. Wierenga CE, Hill $L$, et al. The acceptability, feasibility, and possible benefits of a neurobiologically-informed 5-day multifamily treatment for adults with anorexia nervosa. Int J Eat Disord. 2018;51(8):863-9.

69. Schmidt U, Magill N, et al. The Maudsley outpatient study of treatments for anorexia nervosa and related conditions (MOSAIC): comparison of the Maudsley model of anorexia nervosa treatment for adults (MANTRA) with specialist supportive clinical management (SSCM) in outpatients with broadly defined anorexia nervosa: a randomized controlled trial. J Consult Clin Psychol. 2015;83(4):796-807.

70. Tchanturia K, Davies H, et al. Cognitive remediation therapy for patients with anorexia nervosa: preliminary findings. Ann General Psychiatry. 2007;6:14.

\section{Publisher's Note}

Springer Nature remains neutral with regard to jurisdictional claims in published maps and institutional affiliations.
Ready to submit your research? Choose BMC and benefit from:

- fast, convenient online submission

- thorough peer review by experienced researchers in your field

- rapid publication on acceptance

- support for research data, including large and complex data types

- gold Open Access which fosters wider collaboration and increased citations

- maximum visibility for your research: over $100 \mathrm{M}$ website views per year

At $\mathrm{BMC}$, research is always in progress.

Learn more biomedcentral.com/submissions 